\title{
El bienestar subjetivo a partir de la creatividad y la percepción de felicidad
}

\section{Subjective well-being based on creativity and the perception of happiness}

Dr. Eduardo Ahumada-Tello es profesor e investigador de la Universidad Autónoma de Baja California (México) (eahumada@ uabc.edu.mx) (https://orcid.org/0000-0003-1698-5126)

\begin{abstract}
Resumen
El presente trabajo analiza la relación que existe entre los constructos de «felicidad» y «creatividad» con el «bienestar subjetivo». La importancia de estos elementos se revisa para confirmar la relación positiva que se asume, para de esta manera proponer estrategias de desarrollo organizacional. Los objetivos son encontrar la correlación entre las variables en el entorno local. Para ello se lleva a cabo la aplicación de un cuestionario que incluye ítems para cada una de las variables que conforman el estudio y se obtuvieron 249 respuestas, que son parte de la muestra de una población correspondiente a estudiantes universitarios y a egresados de carreras administrativas en la ciudad de Tijuana, B.C., México. Los resultados confirman que las variables «felicidad» y «bienestar subjetivo» y las variables "creatividad» $\mathrm{y}$ «bienestar subjetivo» presentan una alta correlación con resultados en análisis de correlación de Pearson, en el análisis de varianza y en el modelo de ecuación de regresión las variables. Se concluye la importancia de elaborar estrategias para el desarrollo del elemento humano en las organizaciones a partir de la implantación de acciones que impulsen la creatividad y la percepción de felicidad para que éstas influyan en el incremento del bienestar subjetivo en los individuos y en las organizaciones a partir de redes de colaboración y herramientas organizacionales.
\end{abstract}

\begin{abstract}
In this paper the relationship that exists between the concepts of "happiness» and "creativity" with "subjective wellbeing" is analyzed. The importance of these elements is reviewed to confirm the positive relationship that is supposed to exist between them and in this way to propose organizational development strategies. The goals to achieved are to find the correlation between the variables in the local environment. To this end, a questionnaire is applied, and 249 responses are obtained, this instrument includes questions for each of the variables that are included is the study and have being obtained from a sample of a population of university students and graduates former students of management degrees in the city of Tijuana, BC, Mexico. Results confirm that the variables "happiness» and "subjective wellbeing" and the variables "creativity" and "subjective wellbeing» have a high mutual relationship based on the results in Pearson's correlation analysis, in the analysis of variance and in the regression equation model. It reaches to the conclusion of the importance of applied strategies for the development of the human element in organizations based on the implementation of actions that promote creativity and increase perception of happiness to influence the development of subjective well-being in individuals and organizations from collaboration networks and organizational tools.
\end{abstract}

\section{Palabras claves I keywords}

Bienestar subjetivo, calidad de vida, creatividad, percepción, felicidad, sociedad, organizaciones, individuos. Subjective wellbeing, quality of life, creativity, perception, happiness, society, organizations, people.

Cómo citar: Ahumada-Tello, E. (2019). El bienestar subjetivo a partir de la creatividad y la percepción de felicidad. Retos Revista de Ciencias de la Administración y Economía, 9(18), 327-344. https://doi.org/ 10.17163/ret.n18.2019.09 


\section{Introducción}

\subsection{Antecedentes}

La creatividad y felicidad son temas que se entrelazan cuando se estudian casos organizacionales donde el valor de la empresa depende en gran medida del capital humano. Esta perspectiva atiende la necesidad de contrarrestar la dinámica objetivista que se fundamente en la consecución de objetivos empresariales y organizacionales, sin tomar en cuenta la percepción de los empleados en cuanto a su sentido del bienestar subjetivo, los logros personales y el sentido de pertenencia a la organización (Goucha et al., 2004).

Intentar abordar el tema de la creatividad debe su inicio a la necesidad de explicar una realidad latente sobre su desarrollo. Se puede considerar que la creatividad es parte inherente de la persona desde el inicio de su crecimiento, de tal manera que permanece en ella mientras crece para verse afectada a partir de la introducción de elementos educativos, sociales y culturales en el individuo que regulan sus ideas con las del colectivo social en el que crece y en el que debe de cumplir con requerimientos para su inserción en la sociedad circundante, tanto en aspectos sociales como laborales y económicos. Esto provoca que su creatividad se vea limitada o enfocada primordialmente en aquellos temas que son considerados necesarios en su sociedad para una integración a los sistemas de producción que sustentan el desarrollo económico. Explicar la creatividad ha formado parte de las acciones de investigadores desde finales del siglo pasado. La asociación de «creatividad» $\mathrm{y}$ «felicidad» puede ser considerada una dupla que se presenta de manera natural, puesto que se identifica una como parte de la otra, de manera bidireccional. En este sentido, el constructo de «bienestar subjetivo» se aborda como una consecuencia de la implementación o desarrollo de las dos variables de «felicidad» y de «creatividad» (Chacón, 2005).

A través de diversos estudios se ha hecho mención de cómo la presencia de una percepción depende de la conjugación de diversos factores, tanto sociales como estructurales, en la espera de comprender el fenómeno estudiado. Múltiples factores pueden afectar el desarrollo del bienestar subjetivo. Este está relacionado con los contextos que determinan si la persona es feliz en su área de desarrollo o con las actividades que realiza de manera creativa. Asimismo, se ha estudiado la influencia que se presenta en el optimismo y la autopercepción, como parte consecuente del desarrollo del bienestar subjetivo, asumiendo que al elevarse ambas variables en el individuo, este es capaz de desarrollar un estado interno de mayor satisfacción, y con ello mejorar las relaciones interpersonales positivas (Moreno \& Marrero, 2015).

La literatura sobre «bienestar subjetivo», incluye la felicidad, la satisfacción con la vida y el afecto positivo, se puede revisar en diferentes áreas como la medición, los factores causales y la teoría (Huppert, Baylis \& Keverne, 2012). En términos de influencias causales se encuentran los factores demográficos e influyen la salud, contacto social, actividad y personalidad.

\subsection{Planteamiento del problema}

La creatividad en la organización es parte de una amplia gama de investigaciones en las ciencias médicas y sociales, así como en otros campos científicos relacionados. 
En las últimas cuatro décadas, psicólogos, antropólogos y sociólogos han prestado mayor atención a este constructo. Es posible afirmar que en este momento se sabe más de la creatividad que en cualquier momento de la historia. Este fenómeno no solo forma parte de las artes clásicas como la pintura, música, escultura o literatura, sino que también se enlaza a actividades como la ciencia, el teatro, la innovación empresarial y la creatividad en la resolución de problemas de índole cotidiano.

Además de examinar estudios psicológicos sobre la creatividad y explorar investigaciones sobre felicidad y el bienestar subjetivo, es importante revisar las investigaciones de antropólogos sobre la creatividad en diversas culturas, tanto occidentales como no occidentales; investigaciones sociológicas sobre situaciones, contextos y redes de actividades creativas, así como estudios neurocientíficos cognitivos del cerebro. Todo esto para indagar en los cuestionamientos que comprende la realidad percibida por el individuo y para considerar los contextos sociales y culturales de la creatividad, incluido el papel de la colaboración social y el trabajo en equipo que se requiere para el proceso creativo (Sawyer, 2012).

La felicidad es, por su parte, un factor que ha sido identificado como elemento relevante en el estudio del bienestar subjetivo y de la educación entre estudiantes universitarios, esto desde una perspectiva orientada a la percepción de la capacidad profesional y competitiva de los estudiantes y egresados de programas universitarios (Ahumada-Tello, 2017). Aun así se identifican autores que mencionan que la felicidad tiene características específicas que pueden no hacerla necesariamente parte del bienestar subjetivo (v. gr. Raibley, 2012).

\subsection{Justificación de la investigación}

La «creatividad» y la «felicidad» han sido estudiadas de manera exponencial en los últimos años. Ambos factores son un medio de análisis y evaluación de las estrategias y herramientas que se utilizan para determinar la percepción del «bienestar subjetivo» en los individuos y los grupos sociales (Moccia, 2016). Se entienden entonces como una herramienta de desarrollo organizacional e individual que brinda beneficios personales y profesionales en los diferentes ambientes en los que el individuo se desenvuelve.

La adquisición de nuevas capacidades, experiencias, conocimiento, destrezas, aptitudes y compromisos, así como asumir nuevos retos, metas, objetivos y estatus, es lo que mueve a los individuos a desarrollarse cada vez más, a ser mejores y a proyectarse progresivamente en los cambios del entorno. Esto puede reflejarse de manera perceptiva en el desempeño y en la actitud que ante estos cambios se presenten (Ahumada-Tello, Ravina-Ripoll \& Hernández de Velasco, 2018). Estas actividades pueden ser consideradas como parte de una estrategia para el desarrollo del bienestar subjetivo.

Desde varias perspectivas, la percepción de vivir un bienestar subjetivo puede enfocarse en el contexto del éxito que ha sido un objetivo para alcanzar por los individuos. Es importante hacer mención que el éxito es una escala de procesos y niveles propuestos para una meta. Si el individuo utiliza herramientas que le apoyen a desarrollar conocimiento y ampliar su red de personas a las que conoce, es posible suponer que tendrá un mejor desempeño y en gran medida un mayor grado de satis- 
facción consigo mismo, que se puede equiparar a la felicidad y a un mejor bienestar subjetivo (Huppert et al., 2012).

Con este estudio se podrá determinar la influencia que aporta el constructo de felicidad y el desarrollo de la creatividad a la percepción de un bienestar subjetivo incremental. Esto para mejorar las condiciones que se presentan en los medios que el individuo se desenvuelve personal y profesionalmente. A partir de esta mejoría en la percepción y en su realidad individual y social, es esperable que los resultados profesionales y personales sean mejorados, por lo que es factible que favorezcan y den un resultado efectivo en sus situaciones personales, familiares, sociales, laborales, entre otras y cubriendo con esto las expectativas del entorno (Helliwell \& Putnam, 2004). Esto puede dar cabida a nuevos estudios en diferentes contextos y nuevas variables con las que se quiera explorar el bienestar subjetivo enriqueciendo el conocimiento a través de los diferentes enfoques de investigación

\subsection{Referencias teóricas}

Es importante considerar que el individuo desarrolla el sentido de felicidad partiendo de constructos internos y externos. La ciencia de la felicidad se ha estudiado como un método probado para conseguir el bienestar subjetivo, que puede surgir a través de lo que cada individuo hace y cómo lo hace en los diferentes medios de su desarrollo, pero influenciado por el efecto en los demás y otros que observan sus resultados, convirtiéndose en un estímulo para un buen desempeño en actividades laborales o personales (Lyubomirsky, 2008).

Dentro de estos aspectos, la felicidad personal no solo se puede relacionar con lo objetivo, sino también con el entorno o ambiente en el que se desenvuelven los individuos y que agrega situaciones o factores subjetivos, que pueden ser las relaciones personales, sociales, académicas, laborales y profesionales. Es por esto por lo que las buenas actitudes de conducta se ven reflejados en el trato directo entre los individuos del grupo social y pueden tener la perspectiva de ser positivas o negativas dependiendo del observador que analice este entorno.

La felicidad es una realidad importante en la vida de los individuos. Incluso existe la postura de que la meta de la vida misma es llegar a un punto de felicidad, que de manera filosófica dé sentido a la existencia. La vida de los individuos no tendría sentido sin el sentido de tener una vida feliz. El hombre por su naturaleza busca aquellos retos que lo complementan y le dan metas y posibles logros a su vida, aunque estos sean subjetivos y sujetos a la percepción individual (Angner, 2010). La búsqueda de la felicidad es un objetivo importante para muchas personas y organizaciones. Sin embargo, existe poca investigación científica en el enfoque de obtener y aumentar la felicidad, haciéndola además sostenible y con beneficios económicos permanentes (Easterlin, 2004). Esto es, que se mantenga la percepción a partir de factores internos y externos (Baumeister, Vohs, Aaker \& Garbinsky, 2013).

A pesar de un determinismo pesimista y la adaptación hedónica del destino de la felicidad, existen fuentes emergentes de optimismo con respecto a una felicidad permanente y productiva (Oswald, Proto, \& Sgroi, 2015). Algunos autores indican la existencia de factores que permiten que este tipo de felicidad se sostenga: a) una determinación genética para la felicidad; b) factores circunstanciales externos al indi- 
viduo y; c) actividades y prácticas relevantes para la felicidad individual. Este último elemento o factor es el que el individuo puede controlar e impulsar, es por ello que los esfuerzos de sostenibilidad se deben centrar en este último factor (Lyubomksky, Sheldon \& Schkade, 2005).

La creatividad es una característica básica del ser humano. En ella se reflejan las experiencias y conocimientos del individuo (Ferrando, Prieto, Ferrándiz \& Sánchez, 2005). Existe un incremento en el interés por los estudios en relación con la creatividad debido a su significativo papel en múltiples ámbitos de la ciencia, el arte, la educación y las organizaciones (Pascale, 2005). Esto ha devenido en el surgimiento de modelos y teorías que buscan captar su complejidad y naturaleza multifacética; entre ellos, el modelo de sistemas de Mihaly Csikszentmihalyi, que aparece como un referente mayor en el escenario académico y que propone un importante corrimiento de las posturas tradicionales en psicología, que se centran en el estudio de la creatividad desde la perspectiva de la persona, para pasar a integrar aspectos de la cultura, la sociedad y el individuo en un modelo holístico (Pascale, 2005). Un ejemplo de la aplicación del conocimiento social para el desarrollo de la creatividad son los estudios sobre innovación abierta (Ooms, Bell \& Kok, 2015).

Ésta se encuentra relacionada con la inteligencia. La relación entre creatividad e inteligencia es un tema frecuente de investigación y debate en las Ciencias Sociales. En el caso del trabajo de Sternberg, se examinan las definiciones de creatividad e inteligencia y cómo pueden relacionarse. El modelo desarrollado a partir de su investigación consta de cinco relaciones posibles: a) la creatividad como un subconjunto de inteligencia; b) la inteligencia como subconjunto de la creatividad; c) la creatividad y la inteligencia como conjuntos superpuestos; d) la creatividad y la inteligencia como conjuntos coincidentes; y e) la creatividad y la inteligencia como conjuntos disjuntos. En este sentido, Sternberg aborda la perspectiva de que creatividad e inteligencia deben tener presente una relación mutua que evidencie una coexistencia y dependencia, lo que pone de manifiesto que una no puede existir sin la otra (Plucker, Esping, Kaufman \& Avitia, 2015).

En el ambiente moderno, difícilmente se encuentran casos de personas que por sí mismas lleven a cabo el proceso creativo. Esto sugiere que ahora la creatividad se sustenta en el poder del trabajo en equipo (Zhou, Shin, Brass, Choi \& Zhang, 2009). La existencia de un individuo privilegiado con una creatividad fuera de serie ya no es el único paradigma, sino que se ha visto sustituido por la eficiencia del trabajo multidisciplinario, que es el ambiente presente en los entornos altamente colaborativos en los que operan muchos empresarios y trabajadores.

La creatividad puede ser analizada a través de una lente social para comprender completamente sus aspectos estructurales y relacionales (Cornejo \& Tapia, 2011). En primer lugar, es importante para todas las organizaciones comprender la creatividad desde la perspectiva de una red social y es especialmente relevante para el estudio de la naturaleza empresarial (Moccia, 2016). La creatividad y su investigación desde una perspectiva de red social clasifica los enfoques relacionales y estructurales, donde el primer enfoque enfatiza la fuerza de los vínculos y la calidad de las relaciones y el segundo enfatiza la estructura de las relaciones que lo conforman (Shalley, Hitt, Zhou, Perry-Smith \& Mannucci, 2015). 
El interés por analizar la creatividad desde el enfoque de grupo social tiene que ver con la influencia que se le atribuye a la creatividad e innovación individual, aunque esta visión social no garantiza el éxito (Freire-Gibb \& Nielsen, 2014). En este sentido, la creatividad se puede conceptualizar como un proceso de generación de innovación siguiendo los siguientes pasos: a) generación de la idea; b) elaboración de la idea; c) defensa de la idea; y d) implementación de la idea (Kijkuit \& Van Den Ende, 2007). En este sentido, se han definido como características requeridas para un individuo que desarrolla este proceso las siguientes aplicadas a cada fase anteriormente señalada: a) flexibilidad cognitiva; b) apoyo; c) influencia; y d) visión compartida. Asimismo, se requiere que los creadores individuales cuenten con elementos relacionales y estructurales que les ayuden a evolucionar en cada fase (Perry-Smith \& Mannucci, 2017), siendo importantes también los esfuerzos empresariales para desarrollar e introducir soluciones más automatizadas en los procesos creativos (O’Brien \& Murray, 2015).

En el desarrollo del análisis social de la creatividad se han llevado a cabo estudios que analizan el uso de las redes sociales y su relación con la creatividad de los empleados mediante la adopción de un enfoque de gestión del conocimiento para considerar la influencia de las redes sociales y las interacciones en la creatividad de los individuos (Sigala \& Chalkiti, 2015). El uso más intensivo en tiempo y en actividades de los usuarios que utilizan frecuentemente las redes sociales con especial incidencia en aquellas actividades que permiten mantener contacto y compartir contenidos con sus pares (García, López-de-Ayala \& Catalina, 2013), también ofrecen una oportunidad para documentar, describir y analizar la creatividad (Peppler \& Solomou, 2011), se construyen oportunidades de amistad y colaboración a partir del trabajo en equipo (Gandasegui, 2011), trabajo en equipo en el desarrollo de nuevos productos industriales (Kratzer, Leenders \& Van Engelen, 2010) e ilustrando con acciones conjuntas el futuro de la sociedad (Fundación iS+D, 2011).

Existen propuestas multidimensionales para analizar el bienestar subjetivo en los individuos y grupos sociales (Ryff, 2005). La propuesta de cinco dimensiones de bienestar social, integración social, contribución social, coherencia social, actualización social y aceptación social, está fundamentada teóricamente en la literatura existente (Keyes, 2006).

\section{Materiales y método}

El estudio se realizó a través del enfoque cuantitativo por medio de cuestionarios para determinar si existe una relación entre las variables, así como la recolección de datos para probar la hipótesis planteada. Esto se refleja en datos numéricos y estadísticos para establecer las relaciones existentes. Como investigación no experimental, con alcance correlacional, se busca evaluar la relación que existe entre la variable dependiente e independiente y su comportamiento. 


\section{Figura 1. Diagrama de variables de estudio}

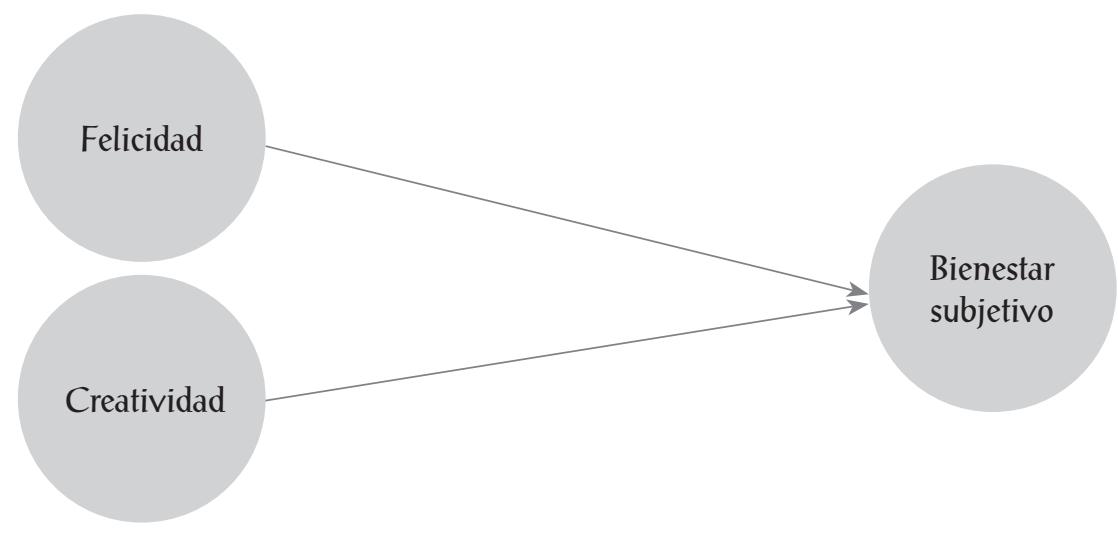

En la Figura 1 se ilustran las variables de estudio que conforman la presente investigación. De esta apreciación se derivan las preguntas de investigación, las hipótesis y los objetivos a cumplir. A continuación, se enumeran estos puntos que serán analizados en los elementos por seguir.

\subsection{Preguntas de investigación}

¿Es la felicidad un factor que influye en la percepción del bienestar subjetivo?

¿Es la creatividad un factor que influye en la percepción del bienestar subjetivo?

\subsection{Hipótesis general}

H. La felicidad y la creatividad son factores que influyen de manera positiva en la percepción del bienestar subjetivo.

H0. La felicidad y la creatividad no son factores que influyen de manera positiva en la percepción del bienestar subjetivo.

\subsection{Hipótesis de investigación}

H1 La felicidad influye de manera positiva en la percepción del bienestar subjetivo. subjetivo.

H10 La felicidad NO influye de manera positiva en la percepción del bienestar

H2 La creatividad influye de manera positiva en la percepción del bienestar subjetivo.

H20 La creatividad NO influye de manera positiva en la percepción del bienestar subjetivo.

\subsection{Objetivo general}

Determinar si la felicidad y la creatividad son factores que influyen de manera positiva en la percepción del bienestar subjetivo. 


\subsection{Objetivos específicos}

- Determinar si la felicidad influye de manera positiva en la percepción del bienestar subjetivo.

- Determinar si la creatividad influye de manera positiva en la percepción del bienestar subjetivo.

Se trata de una investigación aplicada, documental, correlacional, no experimental y transeccional. Se busca con ello la comprensión del fenómeno de investigación, donde se realizó un análisis relativo a la influencia de tres variables: creatividad, felicidad y bienestar subjetivo. Se ha llevado a cabo con estudiantes y egresados de la Facultad de Contaduría y Administración en Tijuana, B.C., México para conocer la precepción sobre las variables de estudio y de la que se sigue el proceso indicado en la figura 2.

\section{Figura 2. Diagrama metodológico}

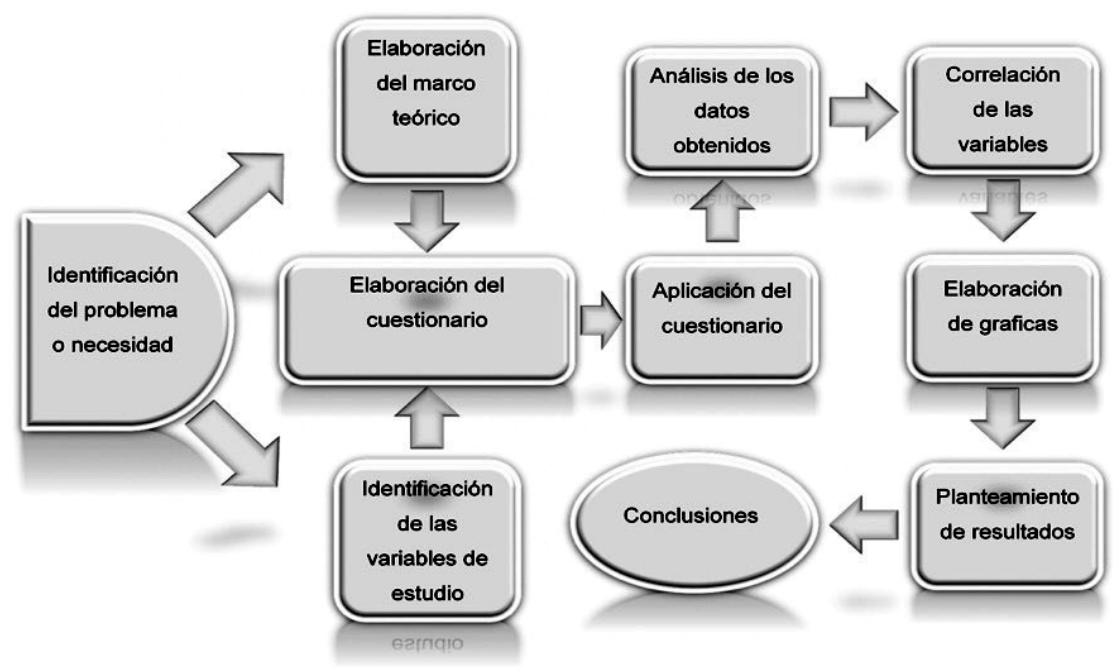

Fuente: Elaboración propia con base en (Hernández-Sampieri, et. al., 2014).

\subsection{Determinación de la muestra}

El número total de cuestionarios se calculó según la fórmula correspondiente a este tipo de muestreo sobre la población de la Facultad de Contaduría y Administración en la Universidad Autónoma de Baja California, que asciende a 3900 alumnos más una población detectada de 1200 egresados, dando por total 5100 individuos para este cálculo. 
Así que, de forma proporcional y con unos niveles de variabilidad iniciales de $\mathrm{p}=\mathrm{q}=0.5$, de confianza del $95 \%$ y un error del $6.21 \%$, para $\mathrm{Z}=1.96$, la muestra fue determinada en 249 instrumentos (Fernández-Nogales, 1998).

Tabla 1. Determinación de la muestra

\begin{tabular}{|l|l|}
\hline \multicolumn{2}{|c|}{ Población Finita } \\
\hline Población (N) & 5,100 alumnos \\
\hline Origen de la población & FCA - UABC \\
\hline P & $50.00 \%$ \\
\hline Q & $50.00 \%$ \\
\hline Error permisible $(\mathrm{E})$ & $6.21 \%$ \\
\hline Z & 1.96 \\
\hline Nivel de confianza $(\mathrm{K})$ & $95.00 \%$ \\
\hline Tamaño de Muestra $(\mathrm{n})=$ & 249 \\
\hline E $=2 *$ raíz $(\mathrm{N}-\mathrm{n}) /(\mathrm{n}-1) *(\mathrm{p} * \mathrm{q} / \mathrm{n})=$ & $6.34 \%$ \\
\hline
\end{tabular}

* Facultad de Contaduría y Administración - Universidad Autónoma de Baja California

\subsection{Descripción de la muestra}

La composición de la muestra de estudio se detalla en la tabla 2, en donde se concentran los datos referentes al género, estado civil, área y nivel de estudios, rango de edades, nacionalidad, país de residencia y actividad laboral. Estos datos nos ofrecen una perspectiva más holística en relación con las características del universo de estudio.

Tabla 2. Datos descriptivos de la muestra

\begin{tabular}{|l|l|l|}
\hline \multicolumn{1}{|c|}{ Indicador descriptivo } & Número & \multicolumn{1}{c|}{ Definición } \\
\hline \multirow{2}{*}{ Género } & 113 & Masculino \\
& 136 & Femenino \\
\hline \multirow{4}{*}{ Estado civil } & 194 & Soltero \\
& 24 & Casado \\
& 22 & Unión libre \\
& 3 & Divorciado \\
& 6 & Otro \\
\hline \multirow{3}{*}{ Edad } & $18-25$ & 197 \\
& $26-35$ & 30 \\
& $36-45$ & 16 \\
& $45-62$ & 6 \\
\hline \multirow{2}{*}{ Nivel de estudios } & 19 & Bachillerato \\
& 209 & Licenciatura \\
& 18 & Maestría \\
& 2 & Doctorado \\
& 1 & Posdoctorado \\
\hline
\end{tabular}




\begin{tabular}{|c|l|l|}
\hline \multicolumn{1}{|c|}{ Indicador descriptivo } & \multicolumn{1}{|c|}{ Definición } \\
\hline Se encuentran laborando & 123 & $\mathrm{Si}$ \\
& 126 & No \\
\hline
\end{tabular}

\subsection{Instrumento de medición}

Para cumplir con los objetivos de la investigación, es necesario operacionalizar el marco teórico y conceptual. Los objetivos y preguntas de investigación, junto con las variables de estudio y las hipótesis, son básicamente los indicadores para construir el instrumento de medición. En este capítulo se procede a aplicar los conocimientos empíricos para recopilar la información de campo. Una vez terminada esta etapa, debe prepararse la información para procesarla. Los puntos que indican este proceso son los siguientes:

- Diseño del instrumento de medición.

- Escalas de medición.

- Pruebas de confiabilidad del instrumento.

- Recolección de datos.

- Procesamiento de datos.

Tabla 3. Escalas del Instrumento de Medición

\begin{tabular}{|l|ll|}
\hline \multicolumn{2}{|c|}{ Tipo de escala } & \multicolumn{1}{c|}{ Amplitud de índice } \\
\hline & 1. & Totalmente en desacuerdo \\
& 2. & En desacuerdo \\
Likert & 3. & Parcialmente en desacuerdo \\
& 4. & Ni de acuerdo ni en desacuerdo \\
& 5. & Parcialmente de acuerdo \\
& 6. & De acuerdo \\
& 7. & Totalmente de acuerdo \\
\hline
\end{tabular}

El instrumento elegido para la recolección de la información fue el cuestionario, siendo este de opciones múltiples a partir de escalas específicas, proporcionando información para el análisis de cada una de las variables que influyen en los factores que intervienen en la percepción del bienestar subjetivo.

Para fines de esta investigación se ha decidido utilizar la escala de Likert para describir las percepciones de los entrevistados en referencia a cada uno de los ítems que conforman el cuestionario. La tabla 3 muestra los valores que se presentan en las respuestas del instrumento y el valor que representan en la columna de amplitud de índice. Finalmente, en la tabla 4 se presenta el instrumento utilizado para llevar a cabo esta investigación 
Tabla 4. Instrumento de Medición

\begin{tabular}{|c|c|c|}
\hline Variables & Ítems & Referencias \\
\hline \multirow{4}{*}{$\begin{array}{l}\text { Bienestar } \\
\text { Subjetivo }\end{array}$} & $\begin{array}{l}\text { El bienestar depende de la percepción } \\
\text { subjetiva que la persona tiene de su } \\
\text { vida. Con respecto a esto, las condi- } \\
\text { ciones de mi vida me hacen sentir to- } \\
\text { talmente realizado. }\end{array}$ & \multirow{4}{*}{$\begin{array}{l}\text { (Angner, 2010), (Atkinson, Bagnall, } \\
\text { Corcoran, South \& Curtis, 2019) } \\
\text { (Goucha et al., 2004), (Moreno \& } \\
\text { Marrero, 2015), (Huppert, Baylis, } \\
\text { \& Keverne, 2012), (Diener \& Ryan, } \\
\text { 2009), (Helliwell \& Putnam, 2004), } \\
\text { (Keyes, 2006), (Nyklíček, Zeelen- } \\
\text { berg, \& Vingerhoets, 2011), (Appau, } \\
\text { Churchill \& Farrell, 2019). }\end{array}$} \\
\hline & $\begin{array}{l}\text { En la mayoría de las cosas que hago, } \\
\text { mi vida está cerca de mi ideal. }\end{array}$ & \\
\hline & $\begin{array}{l}\text { Las actividades y ocupaciones que ten- } \\
\text { go me hacen sentir realizado }\end{array}$ & \\
\hline & $\begin{array}{l}\text { Hasta ahora he conseguido las cosas } \\
\text { que para mí son importantes en la vida }\end{array}$ & \\
\hline \multirow{2}{*}{ Felicidad } & $\begin{array}{l}\text { En general, me considero una } \\
\text { persona feliz }\end{array}$ & \multirow{2}{*}{$\begin{array}{l}\text { (Lyubomirsky, 2008), (Ahumada-Tel- } \\
\text { lo, 2017), (Huppert et al., 2012). } \\
\text { (Baumeister, Vohs, Aaker, \& Garbin- } \\
\text { sky, 2013), (Moccia, 2016) }\end{array}$} \\
\hline & $\begin{array}{l}\text { En comparación con mis compañeros, } \\
\text { me considero una persona feliz }\end{array}$ & \\
\hline \multirow{2}{*}{ Creatividad } & $\begin{array}{l}\text { En términos generales, ¿en qué } \\
\text { medida se considera Ud. una } \\
\text { persona creativa? }\end{array}$ & \multirow{2}{*}{$\begin{array}{l}\text { (Perry-Smith \& Mannucci, 2017), } \\
\text { (Freire-Gibb \& Nielsen, 2014), } \\
\text { (Bourdieu, 2015), (Sawyer, 2012), } \\
\text { (Pascale, 2005), (Ooms, Bell, \& Kok, } \\
\text { 2015), (Zhou, Shin, Brass, Choi, \& } \\
\text { Zhang, 2009), (Chacón, 2005), (Fan- } \\
\text { chini, Jongbloed \& Dirani,(2019) }\end{array}$} \\
\hline & $\begin{array}{l}\text { En términos generales, ¿en qué } \\
\text { medida se considera Ud. una } \\
\text { persona innovadora? }\end{array}$ & \\
\hline
\end{tabular}

\section{Resultados}

\subsection{Pruebas de confiabilidad}

En la Tabla 5 se observa que, al realizar el análisis de confiabilidad de los resultados de la aplicación del cuestionario, se obtienen elevados índices de Alpha de Cronbach, lo que indica que la confiabilidad del instrumento es significativa. También, se puede observar que la relación entre factores y Alpha de Cronbach indica que el instrumento tiene validez de contenido significativo.

Tabla 5. Estudio de Confiabilidad

\begin{tabular}{|l|l|l|}
\hline \multicolumn{1}{|c|}{ Variables } & \multicolumn{1}{c|}{ \# Ítems } & Alpha de Cronbach \\
\hline Bienestar subjetivo & 4 & .877 \\
\hline Creatividad & 2 & .768 \\
\hline Felicidad & 2 & .900 \\
\hline
\end{tabular}

En la Tabla 5, se observan los valores de Alpha de Cronbach para la variable Bienestar Subjetivo es de 0.877, para la variable de Creatividad de 0.768 y para la variable de Felicidad de 0.900 . Los tres valores demuestran que en una proporción 
suficiente, los encuestados comprendieron el mismo constructo para cada uno de los elementos evaluados. De acuerdo con Nunally (1967), cuando este valor es 0.600 o mayor, se considera válido y aceptado para estudios de carácter social.

\subsection{Correlación de Pearson}

Se puede observar en la Tabla 6 una correlación entre Bienestar Subjetivo y Felicidad $(\mathrm{r}=0.672$ y $\mathrm{p}<0.001)$. De la misma manera la correlación entre Bienestar Subjetivo y Creatividad es representativa $(r=0.406$ y $\mathrm{p}<0.001)$. Esta interpretación no implica causalidad, pues la relación solamente implica que las variables covarían entre sí. Es importante considerar que el nivel de correlación en ambos casos es positiva moderada.

Tabla 6. Tabla de correlación de Pearson

\begin{tabular}{|l|l|l|l|l|}
\hline \multicolumn{2}{|c|}{} & $\begin{array}{c}\text { Bienestar } \\
\text { Subjetivo }\end{array}$ & Felicidad & Creatividad \\
\hline \multirow{3}{*}{$\begin{array}{c}\text { Bienestar } \\
\text { Subjetivo }\end{array}$} & Correlación de Pearson & 1 & & \\
\cline { 2 - 6 } & Sig. (bilateral) & & & \\
\cline { 2 - 6 } & $\mathrm{N}$ & 249 & & \\
\hline \multirow{3}{*}{ Felicidad } & Correlación de Pearson & $.672^{* *}$ & 1 & \\
\cline { 2 - 6 } & Sig. (bilateral) & .000 & 249 & \\
\cline { 2 - 6 } & $\mathrm{N}$ & 249 & $.227^{* *}$ & 1 \\
\hline \multirow{3}{*}{ Creatividad } & Correlación de Pearson & $.406^{* *}$ & .000 & 249 \\
\cline { 2 - 6 } & Sig. (bilateral) & .000 & 249 & \\
\cline { 2 - 6 } & $\mathrm{N}$ & 249 & & \\
\hline
\end{tabular}

** La correlación es significativa al nivel 0.01 (bilateral).

Es también importante de mencionar que la correlación entre las variables Felicidad y Creatividad es positiva y representativa, puesto que menciona que $\mathrm{r}=$ 0.227 y confiable puesto que $\mathrm{p}<0.001$. Sin embargo, esta última relación es menor y no indica una alta correlación.

Tabla 7. Evaluación de hipótesis

\begin{tabular}{|l|l|l|}
\hline \multicolumn{1}{|c|}{ Hipótesis } & \multicolumn{1}{|c|}{ Descripción } & \multicolumn{1}{c|}{ Conclusiones } \\
\hline $\mathrm{H}_{1} 0$ & $\begin{array}{l}\text { La felicidad NO influye de manera positiva en } \\
\text { la percepción del bienestar subjetivo }\end{array}$ & Se rechaza porque $\mathrm{p}<.05$ \\
\hline $\mathrm{H}_{2} \mathrm{O}$ & $\begin{array}{l}\text { La creatividad NO influye de manera positiva } \\
\text { en la percepción del bienestar subjetivo }\end{array}$ & Se rechaza porque $\mathrm{p}<.05$ \\
\hline
\end{tabular}

En la Tabla 7 se indica que se rechazan las hipótesis nulas. El análisis de resultados indica que, si existe relación entre las variables con un nivel de significancia aceptable, la correlación entre estas variables es significativa al 0.01 y esto hace que los cambios en una de ellas tiendan a afectar a la otra variable observada en una medida positiva. Es entonces confirmado que la Felicidad y la Creatividad influyen 
de manera positiva y significativa en el Bienestar Subjetivo de la población sujeto de estudio de este trabajo.

\subsection{ANOVA de un Factor}

A continuación, se lleva a cabo el análisis ANOVA para encontrar la relación entre la variable predictora felicidad y la variable dependiente bienestar subjetivo, obteniéndose los resultados que se presentan en las Tablas 8 y 9 .

Tabla 8. ANOVA de un factor I Felicidad - Bienestar Subjetivo

\begin{tabular}{|l|l|l|l|l|l|}
\cline { 2 - 6 } \multicolumn{1}{c|}{} & Suma de cuadrados & df & Media cuadrática & F & Sig. \\
\hline Intragrupos & 753.549 & 22 & 34.252 & 10.038 & 0,000 \\
\hline Entregrupos & 771.150 & 226 & 3.412 & & \\
\hline Total & 1524.699 & 248 & & & \\
\hline
\end{tabular}

En la Tabla 8 se descubre una relación positiva entre el bienestar subjetivo y la felicidad. En este sentido, se advierte la conveniencia de que la percepción de felicidad se debe impulsar y mejorar en la medida de lo posible para impactar sobre el bienestar subjetivo. Los resultados del análisis de varianza indican que la variable predictora posee un impacto significativo sobre el rendimiento académico. Asimismo, los datos de felicidad, donde $\mathrm{F}(22,226)=10.038$ y $\mathrm{p}=0.000$, presenta una tendencia hacia el incremento de bienestar subjetivo conforme se acreciente la percepción del ratio felicidad.

Tabla 9. ANOVA de un factor I Creatividad - Bienestar Subjetivo

\begin{tabular}{|c|l|l|l|l|c|}
\cline { 2 - 6 } \multicolumn{1}{c|}{} & Suma de cuadrados & df & $\begin{array}{c}\text { Media } \\
\text { cuadrática }\end{array}$ & F & Sig. \\
\hline Intragrupos & 394.489 & 22 & 17.931 & 4.030 & 0,000 \\
\hline Entregrupos & 1005.568 & 226 & 4.449 & & \\
\hline Total & 1400.056 & 248 & & & \\
\hline
\end{tabular}

En la Tabla 9 se detenta que hay una relación directa entre el bienestar subjetivo y la creatividad. En este sentido, se advierte la conveniencia de que la creatividad se debe impulsar y mejorar en la medida de lo posible para impactar sobre el bienestar subjetivo. Los resultados del análisis de varianza indican que la variable predictora posee un impacto significativo sobre el rendimiento académico. Asimismo, los datos de felicidad, donde $\mathrm{F}(22,226)=4.030$ y $\mathrm{p}=0.000$, presenta una tendencia hacia el incremento de bienestar subjetivo conforme se acreciente la percepción del ratio felicidad. 
Tabla 10. ANOVA de un factor- Evaluación de Hipótesis

\begin{tabular}{|l|l|c|c|l|}
\hline Hipótesis & \multicolumn{1}{|c|}{ Descripción } & F & p & Conclusiones \\
\hline $\mathrm{H}_{1} 0$ & Felicidad -> Bienestar Subjetivo & $\mathrm{F}(22,226)=10.038$ & 0,000 & Se rechaza \\
\hline $\mathrm{H}_{2} \mathrm{O}$ & $\begin{array}{l}\text { Creatividad -> Bienestar Subje- } \\
\text { tivo }\end{array}$ & $\mathrm{F}(22,226)=4.030$ & 0,000 & Se rechaza \\
\hline
\end{tabular}

En los datos ilustrados en la Tabla 10 se advierte que las hipótesis nulas H10 y H20 se rechazan en correspondencia planteada en el presente artículo. Estos resultados concuerdan con el análisis de correlación que se hicieron previamente mediante el estudio de Pearson. Las pruebas confirman una relación existente entre las variables de estudio.

\subsection{Análisis de regresión}

La regresión múltiple es una técnica estadística a través de la cual se pueden analizar las relaciones entre diferentes variables. Por ejemplo, entre variables dependientes o de criterio y un grupo de variables independientes o predictivas. En la Tabla 11 se ilustra el resultado de aplicar el procedimiento Enter para regresiones lineales. En este caso, se llega a la obtención de un modelo matemático que describe el comportamiento de las variables independientes en relación con la dependiente.

\section{Tabla 11. Coeficientes ${ }^{\mathrm{a}}$}

\begin{tabular}{|l|l|l|l|l|l|l|}
\hline \multirow{2}{*}{ Modelo } & \multicolumn{2}{c|}{$\begin{array}{c}\text { Coeficientes no } \\
\text { estandarizados }\end{array}$} & $\begin{array}{c}\text { Coeficientes } \\
\text { estandarizados }\end{array}$ & \multirow{2}{*}{ t } & \multirow{2}{*}{ Sig. } \\
\cline { 3 - 8 } \multicolumn{2}{|c|}{ B } & \multicolumn{1}{|c|}{ Error Estándar } & \multicolumn{2}{c|}{ Beta } & & \\
\hline \multirow{3}{*}{1} & (Constant) & 3.311 & 1.109 & & 2.985 & .003 \\
\cline { 2 - 8 } & Creatividad & .507 & .086 & .267 & 5.895 & .000 \\
\cline { 2 - 8 } & Felicidad & 1.112 & .082 & .612 & 13.481 & .000 \\
\hline
\end{tabular}

a. Variable Dependiente: BienestarSubjetivo

El modelo obtenido se representa de la siguiente manera en la Tabla 12 donde se muestran los resultados que validan el rechazo de las hipótesis nulas al obtener $\mathrm{P}$ $<0.05$ en todos los elementos que conforman la ecuación de regresión.

\section{Tabla 12. Modelo de regresión}

\begin{tabular}{|l|l|l|l|l|l|}
\hline \multirow{2}{*}{ Modelo } & \multirow{2}{*}{ Constante B } & \multicolumn{2}{|c|}{ Coeficientes } & \multirow{2}{*}{ Método } & $\begin{array}{c}\text { Nivel de } \\
\text { Significancia }\end{array}$ \\
\cline { 3 - 5 } & & Creatividad & Felicidad & & 1.112 \\
\hline 1 & 3.311 & .507 & Enter & $10 \%$ \\
\hline \multicolumn{3}{|c|}{ Bienestar Subjetivo $=\mathbf{3 . 3 1 1}+\mathbf{0 . 5 0 7}$ Creatividad + 1.112 Felicidad } \\
\hline
\end{tabular}


El modelo evidencia que la felicidad tiene una mayor influencia en el sentido de percepción de bienestar subjetivo que la creatividad. Este análisis confirma los resultados de la correlación de Pearson y el Análisis de Varianza.

\section{Conclusiones y discusión}

La felicidad y la creatividad son parte de la vida del ser humano. En ambos constructos, ocurren fenómenos que los potencializan o los restringen. Es, en este sentido, que la importancia del estudio de estas variables en relación con el bienestar subjetivo se vuelve relevante. La percepción de una emoción es variable y se encuentra sujeta a un sinnúmero de factores que afectan a los individuos ante la prerrogativa de indicar un nivel de evaluación para conceptos basados en apreciación. El hecho de relacionar los constructos de «felicidad» $\mathrm{y}$ "creatividad» con el «bienestar subjetivo» abre el análisis de los escenarios donde estos factores iniciales son promovidos y finalmente aceptados como parte de la sensación de bienestar subjetivo, al que los individuos aspiran y puedan acceder de acuerdo con estrategias que se impulsen en las áreas laborales y educativas.

En una sociedad que evoluciona y se transforma de manera constante, el factor humano se convierte en el elemento fundamental para el crecimiento socioeconómico. Es por ello por lo que indagar en los constructos que afectan su percepción de satisfacción, y por ende una apreciación del bienestar subjetivo, se vuelve importante. Las organizaciones y la sociedad en general deben estar enfocadas en lograr sus metas sociales y económicas apoyándose en un elemento humano satisfecho con sus logros y consciente de sus limitaciones en el ámbito personal y social. Estudios sobre estos conceptos subjetivos abren la posibilidad de comprender de mejor manera qué es lo que hace que un individuo considere como parte de su realización y en qué nivel esto afecta sus percepciones personales.

En esta investigación se aplicaron un total de 249 cuestionarios. Las variables objetos del presente estudio se analizaron por medio de las técnicas estadísticas del Alfa de Cronbach, correlación de Pearson, análisis de varianza y ecuación de regresión. Los resultados obtenidos ponen de manifiesto los siguientes hallazgos:

- La hipótesis $\mathrm{H}^{1}$ que indica que a mayor felicidad habrá mejor bienestar subjetivo en estudiantes y egresados de la Universidad Autónoma de Baja California se valida en las pruebas estadísticas de correlación de Pearson, el análisis de varianza y la ecuación de regresión. Esto confirma de manera dual la relevancia de la felicidad en la percepción de un mejor bienestar subjetivo de los estudiantes y graduados (Ravina-Ripoll, Galiano-Coronil \& Tobar-Pesántez, 2019).

- La hipótesis $\mathrm{H}^{2}$ que hace referencia a la creatividad como factor de impacto en el bienestar subjetivo de los estudiantes y egresados de la Universidad Autónoma de Baja California se ratifica en la prueba de correlación de Pearson, el análisis de varianza, así como el análisis de regresión, por esta razón se acepta y como resultado final de la investigación se 
considera que se cumplen los requisitos para su aceptación confirmando el ejercicio de correlación donde ya ha sido aceptada.

Los resultados encontrados confirman la necesidad de ampliar el espectro de variables a atender para comprender el fenómeno de incrementar el bienestar subjetivo acorde a su naturaleza subjetiva (Diener \& Ryan, 2009). Como se puede confirmar, la felicidad si es importante para el bienestar subjetivo al igual que la creatividad. Es por ello por lo que en trabajos futuros es conveniente realizar abordajes con características holísticas, por ejemplo, incluir las diferencias en sistemas económicos, las cuestiones sociales, culturales y emocionales (Nyklíček, Zeelenberg \& Vingerhoets, 2011), la influencia de las tendencias modernas en cuanto a equidad de género y modificación o consolidación de valores individuales y sociales que refuercen la percepción de felicidad, las estrategias de creatividad y por consiguiente el bienestar subjetivo.

Este tema de estudio es amplio y de igual manera es importante para comprender el comportamiento hacia la obtención de un crecimiento organizacional. Se espera que el bienestar subjetivo sea un precedente de lo que en el futuro será la capacidad de resolución de problemas y cumplimiento de objetivos en el ámbito laboral. Inclusive ir más allá y considerar que las conductas tendientes hacia buenos resultados se pueden extrapolar al éxito en las relaciones personales.

\section{Referencias}

Ahumada-Tello, E. (2017). Perception of Personal Competitiveness. A Study from the Perspective of Happiness, Well-Being and Education in University Graduates. Revista de Estudios Empresariales - Segunda Epoca, (1), 34-54. https://doi.org/ 10.17561/ree.v0i1.3187

Ahumada-Tello, E., Ravina-Ripoll, R., \& Hernández de Velasco, J. (2018). El rol de las redes digitales en el desempeño académico y en la percepción de felicidad social en estudiantes universitarios en Baja California. En R. Ravina Ripoll, L. Tobar y A. Galiano (Coords.), Claves para un desarrollo sostenible: la creatividad y el" happiness management" como portafolio de la innovación tecnológica, empresarial y marketing social (pp. 99-116). España: Editorial Comares.

Angner, E. (2010). Subjective well-being. Journal of Socio-Economics, 39(3), 361-368. https://doi. org/10.1016/j.socec.2009.12.001

Appau, S., Churchill, S.A., \& Farrell, L. (2019). Social integration and subjective wellbeing. Applied Economics, 51(16), 1748-1761. https://doi.org/10.1080/00036846.2018.1528340

Atkinson, S., Bagnall, A.M., Corcoran, R, South, J., \& Curtis, S. (2019). Being Well Together: Individual Subjective and Community Wellbeing. Journal of Happiness Studies, Article in Press, 1-19. https://doi.org/10.1007/s10902-019-00146-2

Baumeister, R. F., Vohs, K. D., Aaker, J. L., \& Garbinsky, E. N. (2013). Some key differences between a happy life and a meaningful life. Journal of Positive Psychology, 8(6), 505-516. https:// doi.org/10.1080/17439760.2013.830764

Chacón, Y. (2005). Una revisión crítica del concepto de creatividad. Actualidades Investigativas n Educación, 5(1), 1-30. https://doi.org/10.15517/aie.v5i1.9120

Cornejo, M., \& Tapia, M. L. (2011). Redes sociales y relaciones interpersonales en internet. Fundamentos En Humanidades, 12(2), 219-229.

Diener, E., \& Ryan, K. (2009). Subjective well-being: A general overview. South African Journal of Psychology, 39(4), 391-406. https://doi.org/10.1177/008124630903900402

Easterlin, R. A. (2004). The economics of happiness. Daedalus, 133(2), 26-33. https://doi. org/10.1162/001152604323049361 
Fanchini, A., Jongbloed, J., \& Dirani, A. (2019). Examining the well-being and creativity of schoolchildren in France. Cambridge Journal of Education, 49(4), 391-416. https://doi.org/10.1 080/0305764X.2018.1536197

Fernández-Nogales, A. (1998). Investigación de mercados: obtención de información. Madrid, España: S.L. CIVITAS EDICIONES.

Ferrando, M., Prieto, M. D., Ferrándiz, C., \& Sánchez, C. (2005). Inteligencia y creatividad. Electronic Journal of Research in Educational Psychology, 3(3), 21-49.

Freire-Gibb, L. C., \& Nielsen, K. (2014). Entrepreneurship Within Urban and Rural Areas: Creative People and Social Networks. Regional Studies, 48(1), 139-151. https://doi.org/10.1080 /00343404.2013.808322

Gandasegui, V. (2011). Mitos y realidades de las redes sociales. Prisma Social: Revista de Ciencias Sociales, (6), 1-26.

García, A., López-de-Ayala, M., \& Catalina, B. (2013). Hábitos de uso en Internet y en las redes sociales de los adolescentes españoles. Revista Científica de Comunicación y Educación, 21(41), 195-204.

Gaut, B. (2010). The Philosophy of Creativity. Philosophy Compass, 5(12), 1034-1046. https://doi. org/10.1111/j.1747-9991.2010.00351.x

Helliwell, J. F., \& Putnam, R. D. (2004). The social context of well-being. En Philosophical Transactions of the Royal Society B: Biological Sciences, 359(1449). https://doi.org/10.1098/rstb.2004.1522

Hernández-Sampieri, R., Fernández-Collado, C., \& Baptista-Lucio, P. (2014). Metodología de la Investigación (6ta. ed.). México: McGraw-Hill.

Huppert, F. A., Baylis, N., \& Keverne, B. (2012). The Science of Well-Being. United Kingdom: Oxford Scholarship Online. https://doi.org/10.1093/acprof:oso/9780198567523.001.0001

Keyes, C. L. M. (2006). Social Well-Being. Social Psychology Quarterly, 61(2), 121-140 https://doi. org/10.2307/2787065

Kijkuit, B., \& Van Den Ende, J. (2007). The organizational life of an idea: Integrating social network, creativity and decision-making perspectives. Journal of Management Studies, 44(6), 865-882. https://doi.org/10.1111/j.1467-6486.2007.00695.x

Kratzer, J., Leenders, R. T. A. J., \& Van Engelen, J. M. L. (2010). The social network among engineering design teams and their creativity: A case study among teams in two product development programs. International Journal of Project Management, 28(5), 428-436. https://doi. org/10.1016/j.ijproman.2009.09.007

Lyubomirsky, S. (2008). La ciencia de la felicidad: un método probado para conseguir el bienestar. Barcelona, España: Ediciones Urano.

Lyubomksky, S., Sheldon, K. M., \& Schkade, D. (2005). Pursuing happiness: The architecture of sustainable change. Review of General Psychology, 9(2), 111-131. https://doi.org/10.1037/10892680.9.2.111

Moccia, S. (2016). Felicidad en el Trabajo. Papeles Del Psicólogo, 37(2), 143-151.

Moreno, Y., \& Marrero, R. (2015). Optimismo y autoestima como predictores de bienestar personal: diferencias de género. Revista Mexicana de Psicología, 31(1), 27-36.

Nunally, J. C. (1967). Psychometric Theory. New York, Estados Unidos: McGraw Hill.

Nyklíček, I., Zeelenberg, M., \& Vingerhoets, A. (2011). Emotion regulation and well-being. New York, Estados Unidos: Springer. https://doi.org/10.1007/978-1-4419-6953-8

O’Brien, C. \& Murray, S.E. (2015). Sustainable wellbeing, creativity and innovation. International Journal of Innovation, Creativity and Change, 2(1), 117-126.

Ooms, W., Bell, J., \& Kok, R. A. W. (2015). Use of Social Media in Inbound Open Innovation: Building Capabilities for Absorptive Capacity. Creativity and Innovation Management, 24(1), 136-150. https://doi.org/10.1111/caim.12105

Oswald, A. J., Proto, E., \& Sgroi, D. (2015). Happiness and Productivity. Journal of Labor Economics, 33(4), 789-822. https://doi.org/10.1086/681096 
Pascale, P. (2005). ¿Dónde está la creatividad? Una aproximación al modelo de sistemas de Mihaly Csikszentmihalyi. Arte Individuo y Sociedad, 17, 63-86.

Peppler, K. A., \& Solomou, M. (2011). Building creativity: collaborative learning and creativity in social media environments. On the Horizon, 19(1), 13-23. https://doi. org/10.1108/10748121111107672

Perry-Smith, J. E., \& Mannucci, P. V. (2017). From creativity to innovation: The social network drivers of the four phases of the idea journey. Academy of Management Review, 42(1). https:// doi.org/10.5465/amr.2014.0462

Plucker, J. A., Esping, A., Kaufman, J. C., \& Avitia, M. J. (2015). Creativity and intelligence. En S. Goldstein, D. Princiotta, y J. A. Naglieri (Eds.), Handbook of Intelligence: Evolutionary Theory, Historical Perspective, and Current Concepts (283-291). New York, Estados Unidos: Springer. https://doi.org/10.1007/978-1-4939-1562-0_19

Raibley, J. R. (2012). Happiness is not Well-Being. Journal of Happiness Studies, 13(6), 1105-1129. https://doi.org/10.1007/s10902-011-9309-z

Ravina-Ripoll, R., Galiano-Coronil, A., \& Tobar-Pesántez, L. (2019). Towards A Happy, Creative And Social Higher Education Institution: The Case Of Non-Profit Marketing And Business Creation Subjects At The University Of Cadiz. Journal of Entrepreneurship Education, 22(1), 1-8. Recuperado de https://bit.ly/2MPXQ60

Ryff, C. D. (2005). Happiness is everything, or is it? Explorations on the meaning of psychological well-being. Journal of Personality and Social Psychology, 57(6), 1069-1081. https://doi. org/10.1037/0022-3514.57.6.1069

Sawyer, R. K. (2012). Explaining Creativity: The Science of Human Innovation. Creativity. New York, Estados Unidos: Oxford University Press.

Perry-Smith, J., \& Mannucci, P. V. (2015). Social Networks, Creativity, and Entrepreneurship. En C. E. Shalley, M. A. Hitt, \& J. Zhou (Eds.), The Oxford Handbook of Creativity, Innovation, and Entrepreneurship. Oxford Handbooks Online. https://doi.org/10.1093/oxford$\mathrm{hb} / 9780199927678.013 .0016$

Sigala, M., \& Chalkiti, K. (2015). Knowledge management, social media and employee creativity. International Journal of Hospitality Management, 45, 44-58. https://doi.org/10.1016/j. ijhm.2014.11.003

Smith, T.S.J., \& Reid, L. (2018). Which 'being' in wellbeing? Ontology, wellness and the geographies of happiness. Progress in Human Geography, 42(6), 807-829. https://doi. org/10.1177/0309132517717100

Zhou, J., Shin, S. J., Brass, D. J., Choi, J., \& Zhang, Z. X. (2009). Social Networks, Personal Values, and Creativity: Evidence for Curvilinear and Interaction Effects. Journal of Applied Psychology, 94(6), 1544-1552. https://doi.org/10.1037/a0016285 